\title{
Psoriasis and Cardiometabolic Comorbidities: An Evaluation of the Impact of Systemic Treatments in Randomized Clinical Trials
}

\author{
Megan Mosca (D) · Julie Hong · Edward Hadeler · Marwa Hakimi • \\ Nicholas Brownstone · Wilson Liao · Tina Bhutani
}

Received: June 11, 2021 / Accepted: August 6, 2021 / Published online: September 10, 2021

(C) The Author(s) 2021

\section{ABSTRACT}

The association between psoriasis, metabolic syndrome, and cardiovascular disease is well established. The shared pathways between psoriasis, metabolic syndrome, and atherosclerosis suggest that treatments targeting the inflammatory pathways of psoriasis may also be beneficial in the treatment of associated cardiometabolic comorbidities. This paper reviews the most recent data regarding the impact of systemic psoriasis treatments on comorbid cardiovascular and metabolic disease. Data from randomized clinical trials with systemic and biologic agents are presented. Overall, studies demonstrate beneficial effects on several cardiometabolic markers and risk factors in psoriasis patients; however, longer randomized controlled trials to characterize the direct benefit for cardiovascular outcomes are needed.

Keywords: Psoriasis; Cardiovascular disease; Metabolic syndrome; Systemic; Biologic

M. Mosca $(\bowtie) \cdot$ J. Hong · E. Hadeler · M. Hakimi .

N. Brownstone $\cdot$ W. Liao $\cdot$ T. Bhutani

Department of Dermatology, University of

California San Francisco, Psoriasis and Skin

Treatment Center, 515 Spruce Street, San Francisco,

CA 94118, USA

e-mail: Megan.mosca@ucsf.edu

\section{Key Summary Points}

Cardiometabolic comorbidities are highly prevalent in psoriasis patients.

Systemic agents targeting common immune pathways between psoriasis, cardiovascular disease, and metabolic syndrome have the potential to positively impact all three health conditions.

Adalimumab, methotrexate, cyclosporine, etanercept, infliximab, secukinumab, tofacitinib, and ustekinumab were associated with varying levels of impact on the imaging and biomarkers of cardiometabolic disease in psoriasis patients when evaluated in randomized clinical trials.

Based on the available data, it may be beneficial to consider early, sustained systemic therapy in psoriasis patients at an elevated risk of cardiovascular disease.

\section{INTRODUCTION}

Psoriasis is a chronic, immune-mediated, inflammatory skin condition that affects over 100 million people worldwide [1]. For many 
years, psoriasis was thought to be a disease that primarily affects the skin and joints, but its association with cardiovascular and metabolic disorders is now widely studied and documented. In fact, there is growing evidence to suggest that psoriasis may be an independent risk factor for cardiovascular disease [2, 3]. These patients are at increased risk of cardiovascular risk factors such as metabolic syndrome and its individual components (obesity, dyslipidemia, hypertension, and insulin resistance) compared to patients without psoriasis $[2,4,5]$. While the exact mechanism behind this relationship is unclear, common underlying pathways of systemic inflammation mediated by type 1 helper (Th1) and type 17 helper (Th17) cells, endothelial dysfunction, and pathologic angiogenesis may explain the link between the pathogenesis of psoriasis and cardiometabolic diseases [6, 7].

Cardiometabolic comorbidities negatively impact multiple domains of the lives of psoriasis patients, resulting in increased morbidity and mortality. The presence of comorbid cardiovascular disease not only negatively impacts quality of life; it is also significantly associated with impairment of work and physical activity and with greater healthcare utilization [8]. The added financial stress induced by increased medical costs, office visits, and hospitalizations also plays a role in the overall burden placed on psoriasis patients with comorbid disease. Furthermore, patients with severe psoriasis are at an increased risk of cardiovascular mortality even after accounting for traditional risk factors [hazard ratio (HR) 1.57, 95\% confidence interval (CI) 1.26-1.96] [9].

The substantial burden associated with cardiometabolic comorbidities is a motivating factor for exploring treatments that may lead to additional benefits beyond skin clearance. The shared pathogenic pathway between psoriasis and cardiometabolic disease suggests that treatments which target the systemic inflammation of psoriasis may also have the added benefit of improving associated cardiometabolic comorbidities and risk factors. In fact, previous retrospective studies have shown a decrease in the development of cardiovascular disease following treatment with systemic agents [10].
Data from randomized clinical trials provide further support for the direct and indirect impacts of systemic therapy for psoriasis on cardiovascular disease and metabolic dysfunction. Detailed below is the evidence from randomized clinical trials of the impacts of tumor necrosis factor (TNF)- $\alpha$ inhibitors, interleukin (IL)-17, IL-12/23, and other systemic agents on cardiovascular risk factors and biomarkers in psoriasis patients.

\section{METHODS}

A literature search of PubMed for randomized clinical trials through 3 May 2021 with the terms "psoriasis" AND ("cardiovascular" OR "metabolic syndrome" OR "diabetes" OR "hypertension" OR "dyslipidemia" OR "hyperlipidemia" OR "obesity") AND ("systemic" OR "biologic") was conducted. Studies were excluded for the following reasons: an observational study design without subject randomization, no active comparator, primary outcomes that did not assess cardiometabolic parameters, or because no plaque psoriasis patients were included in the study. Comprehensive review articles were referenced to identify any additional studies that were missed. Twelve randomized clinical trials that evaluate the effects of systemic therapies in plaque psoriasis patients on cardiovascular or metabolic risk factors and biomarkers were identified. This article is based on previously conducted studies and does not contain any new studies with human participants or animals performed by any of the authors.

\section{RESULTS}

The significant findings of the randomized clinical trials are reviewed below. Table 1 provides additional details and expands on the significant results of each study.

\section{TNF- $\alpha$ inhibitors}

Several studies have evaluated the cardiometabolic impact of TNF- $\alpha$ inhibitor agents 


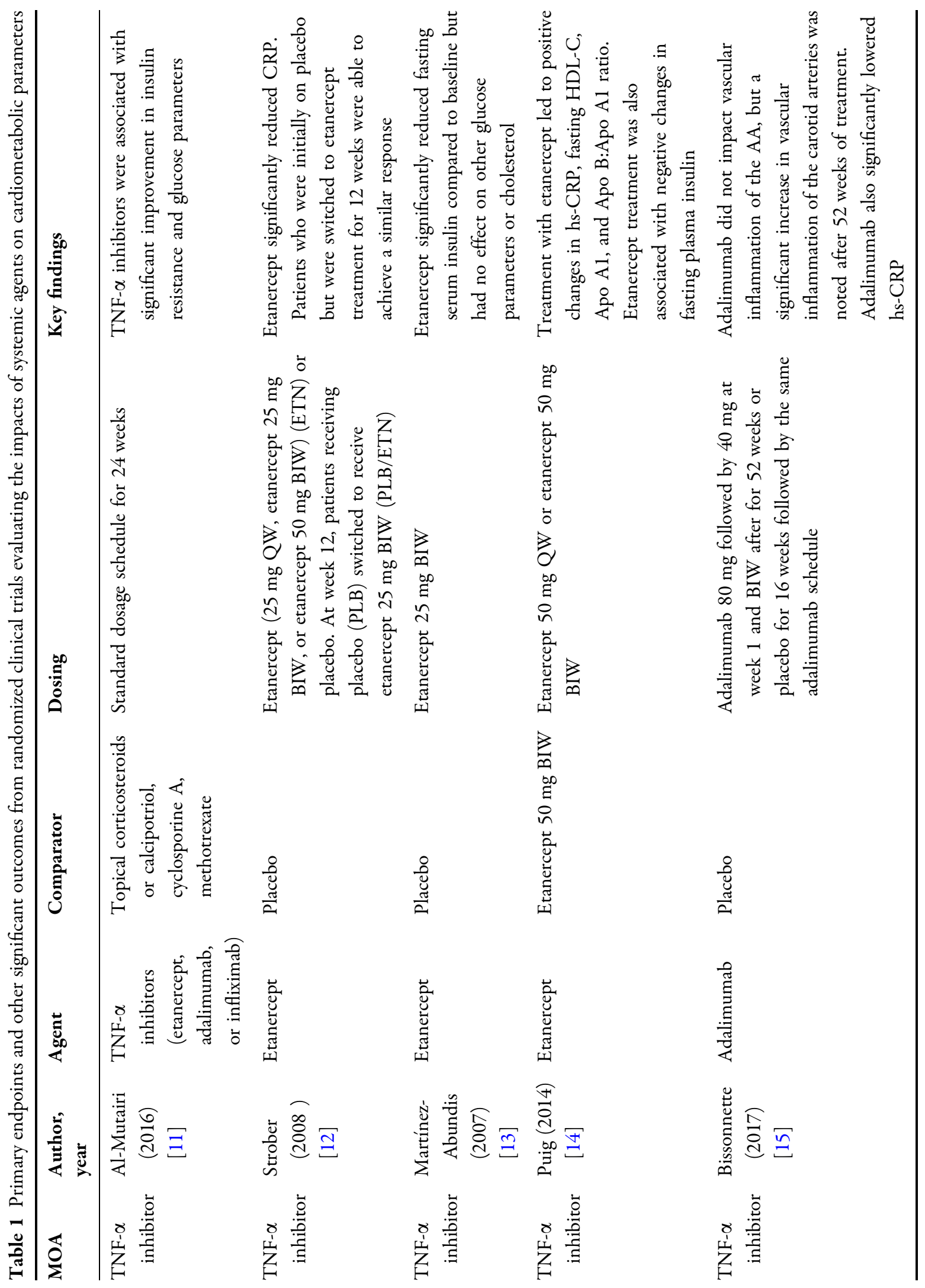




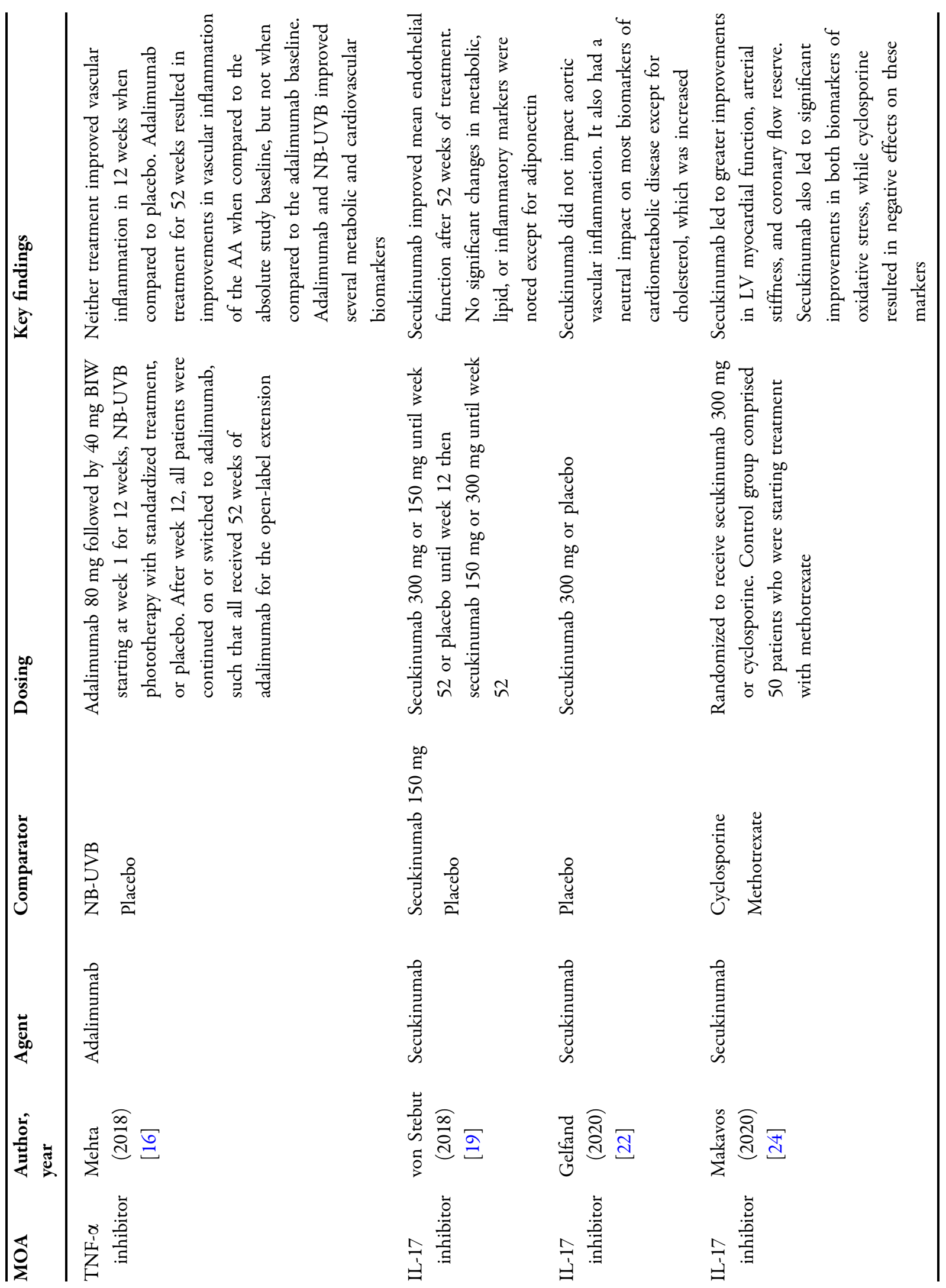




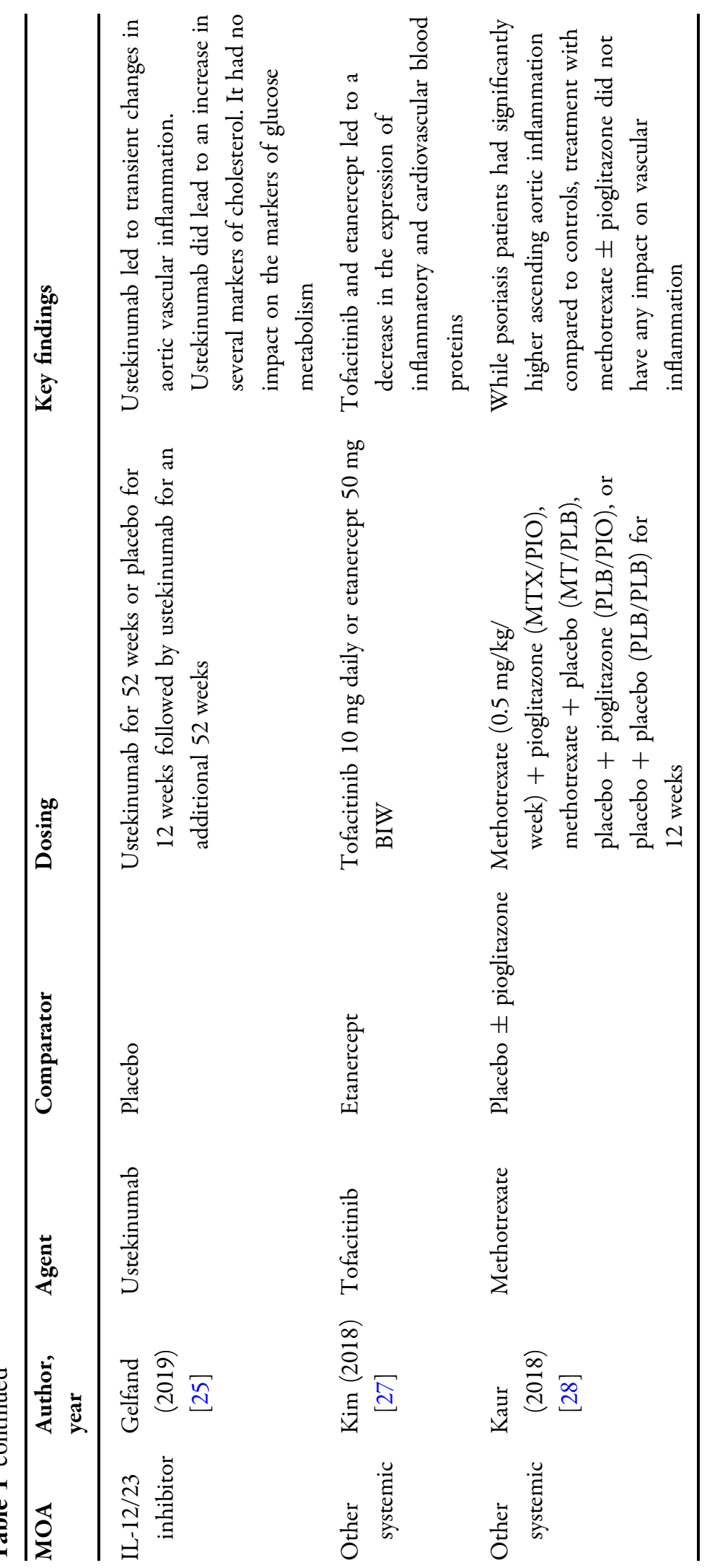




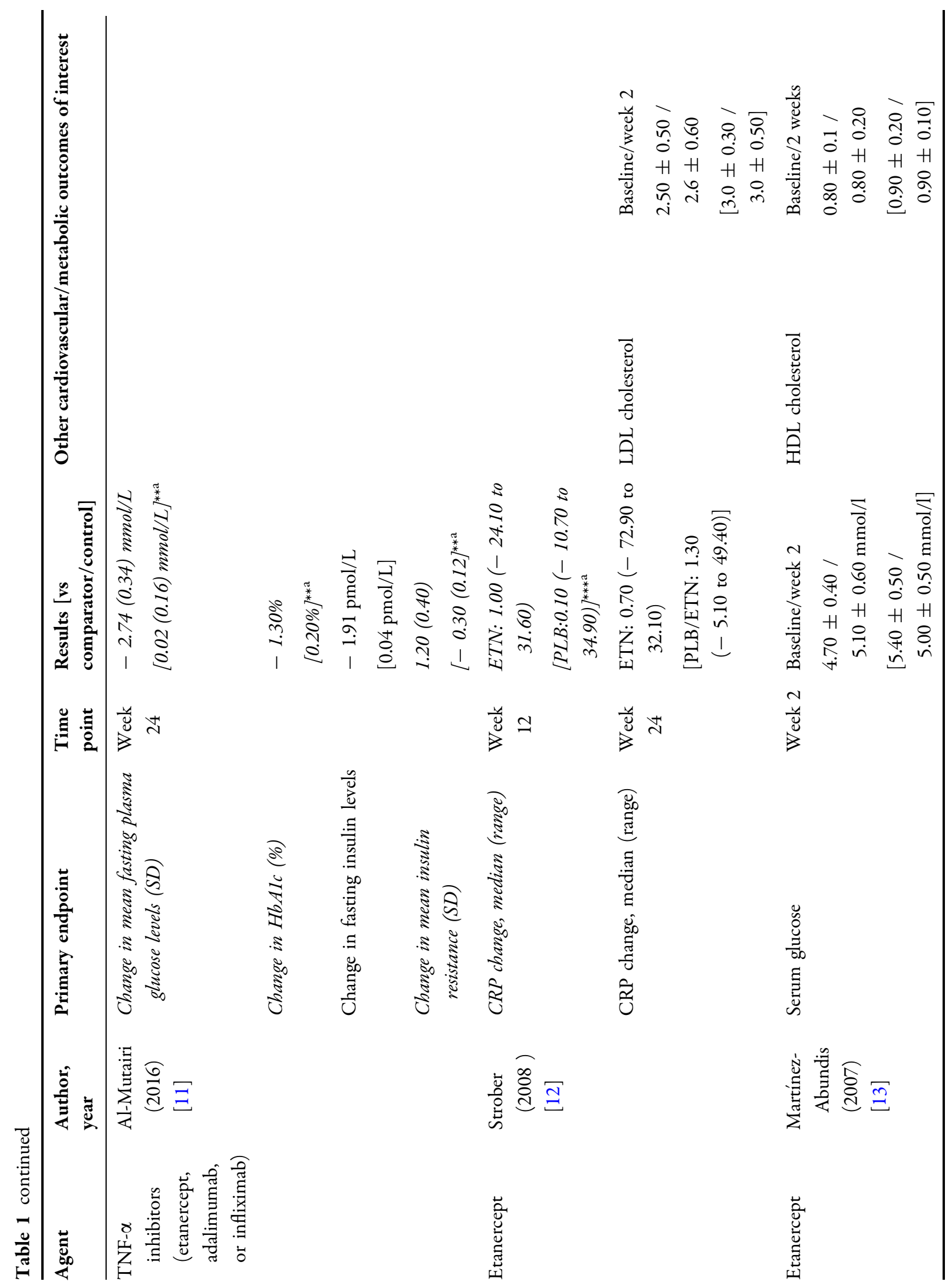




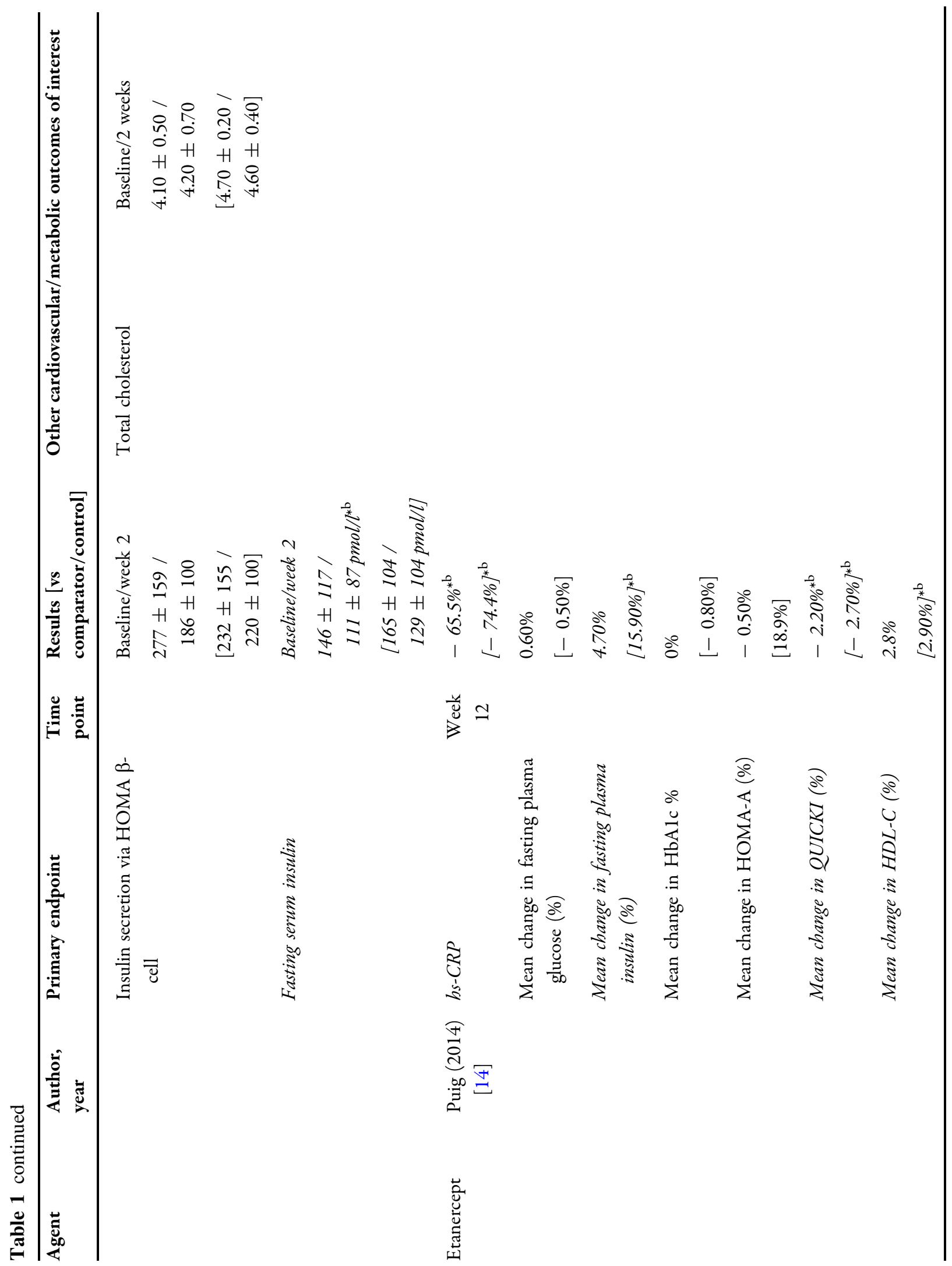




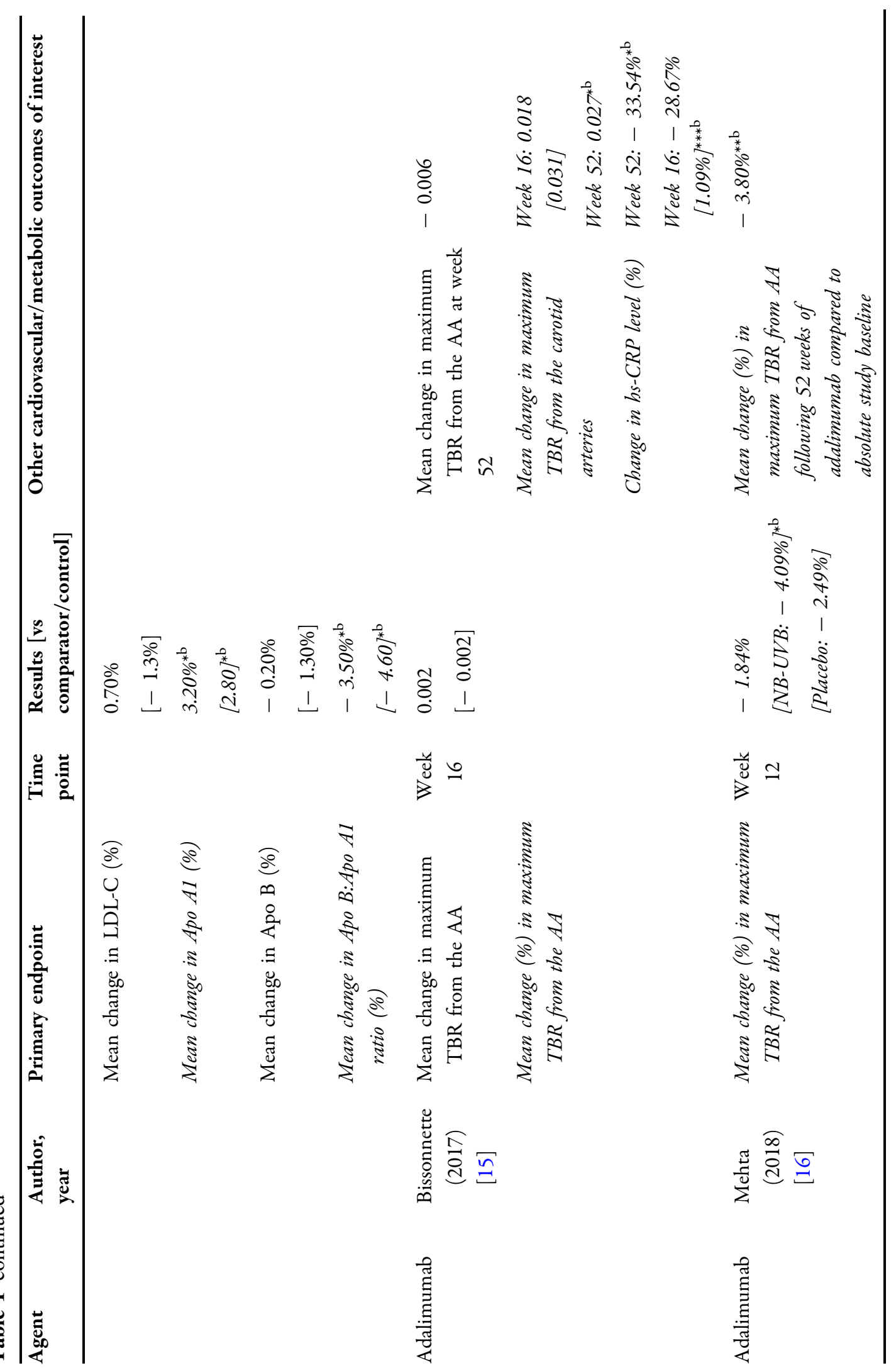




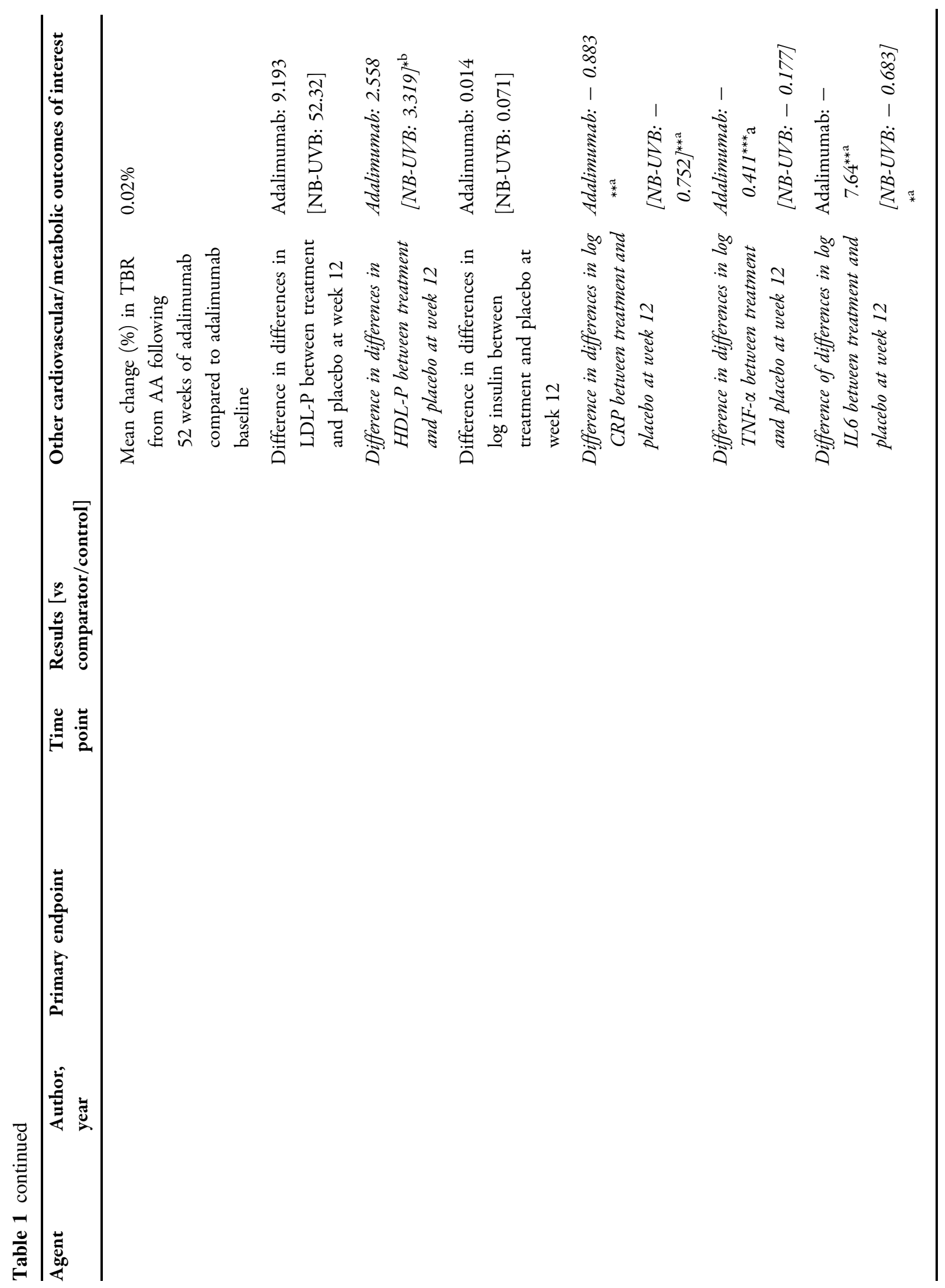




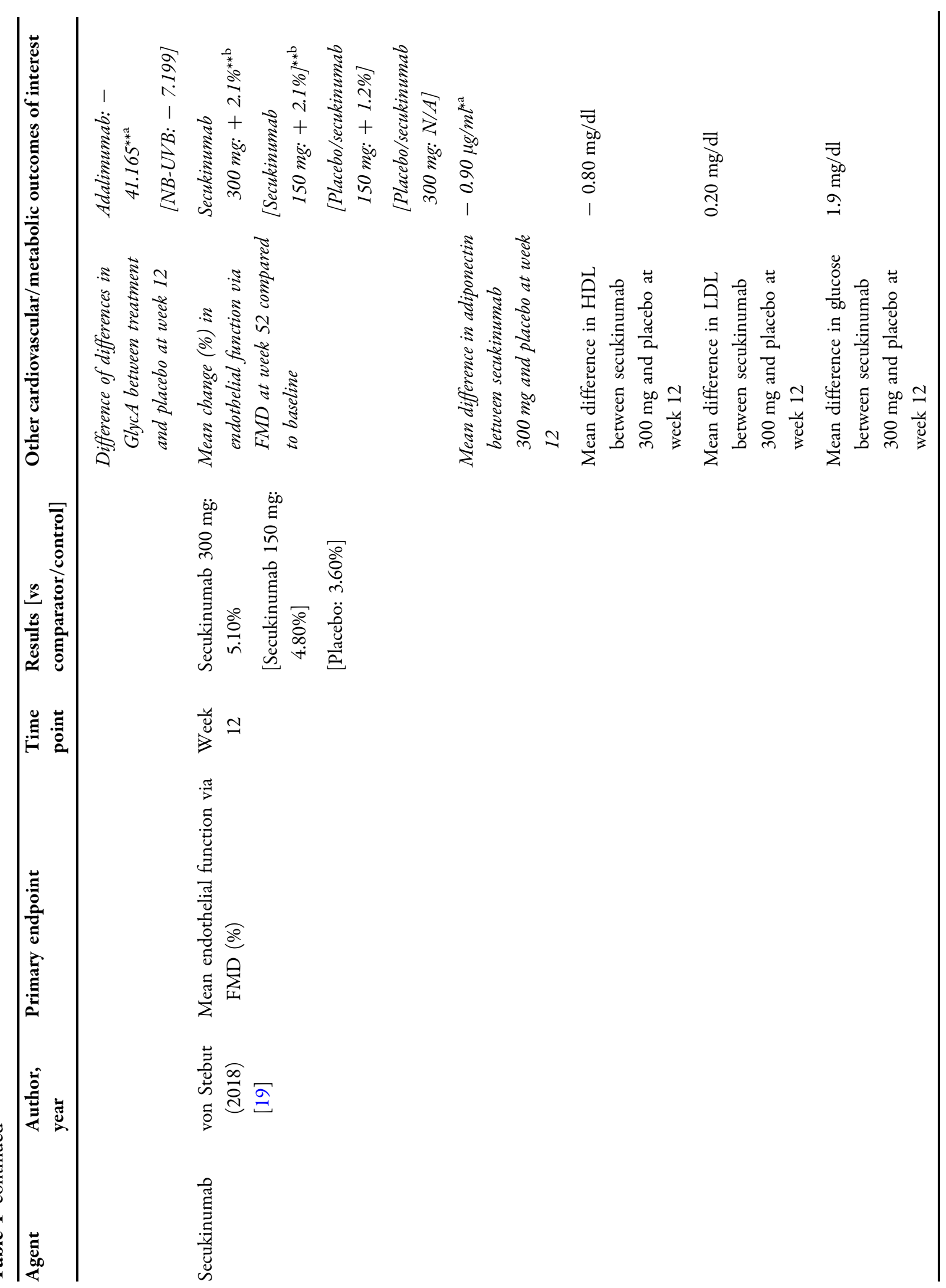




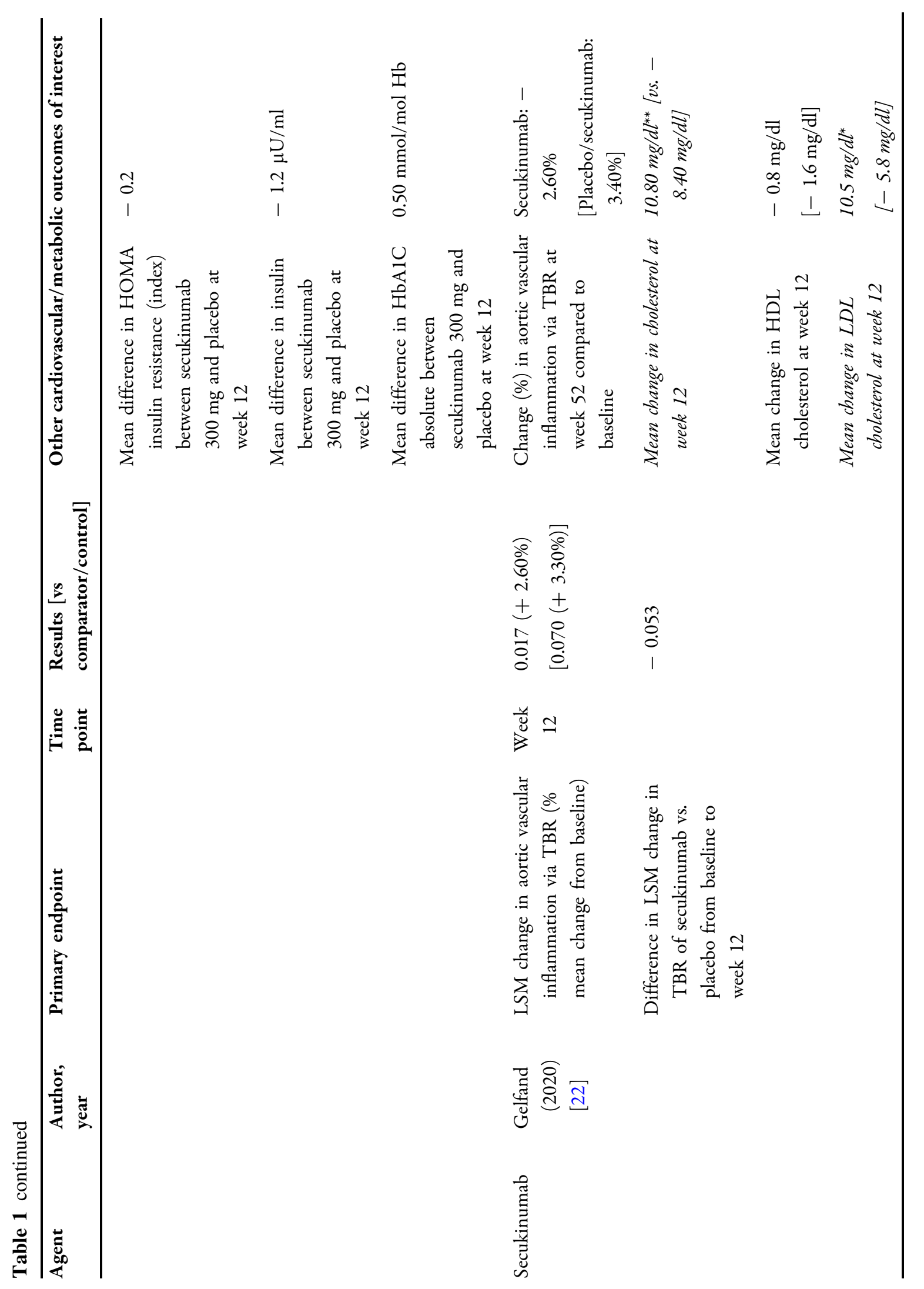




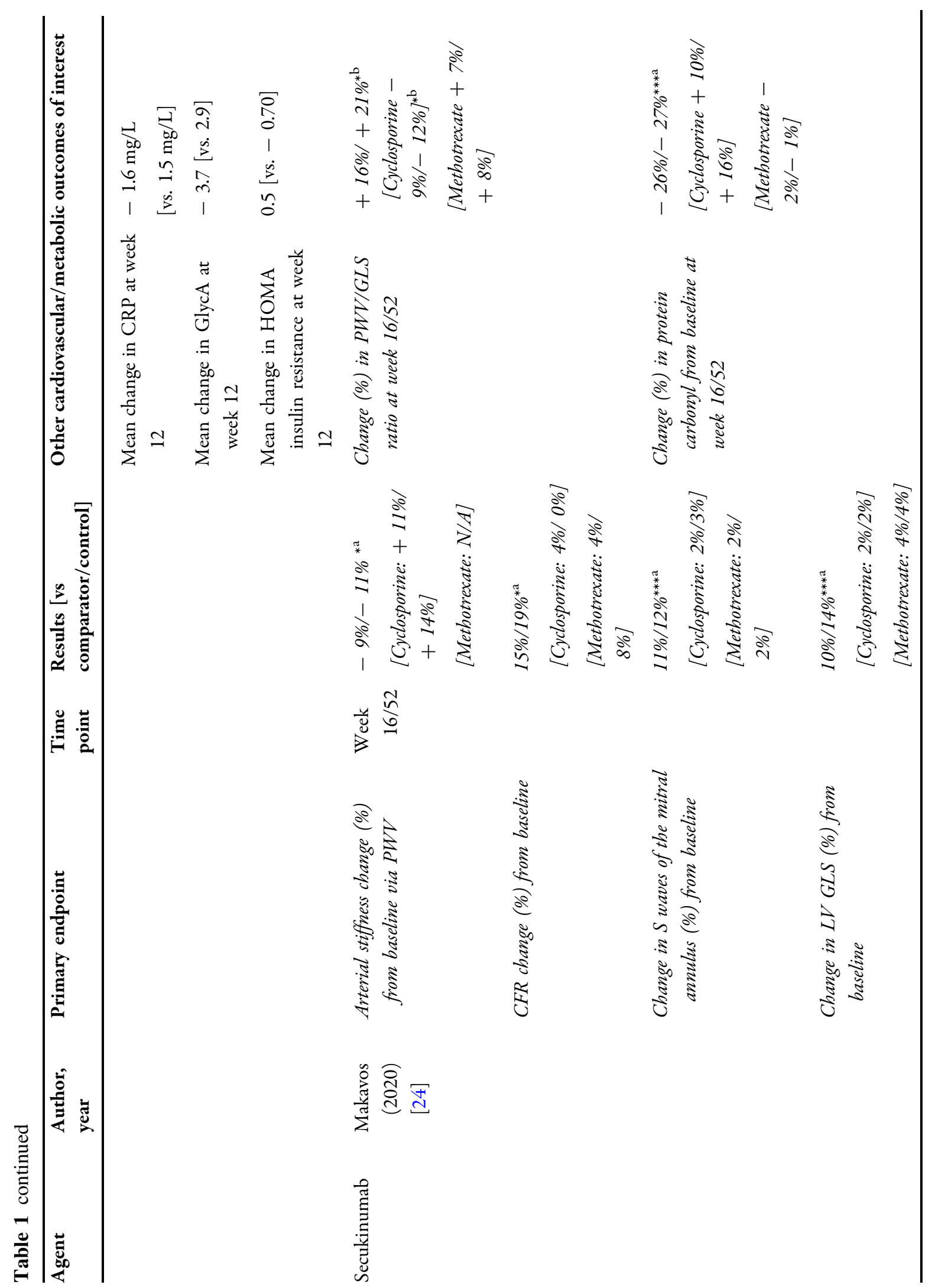




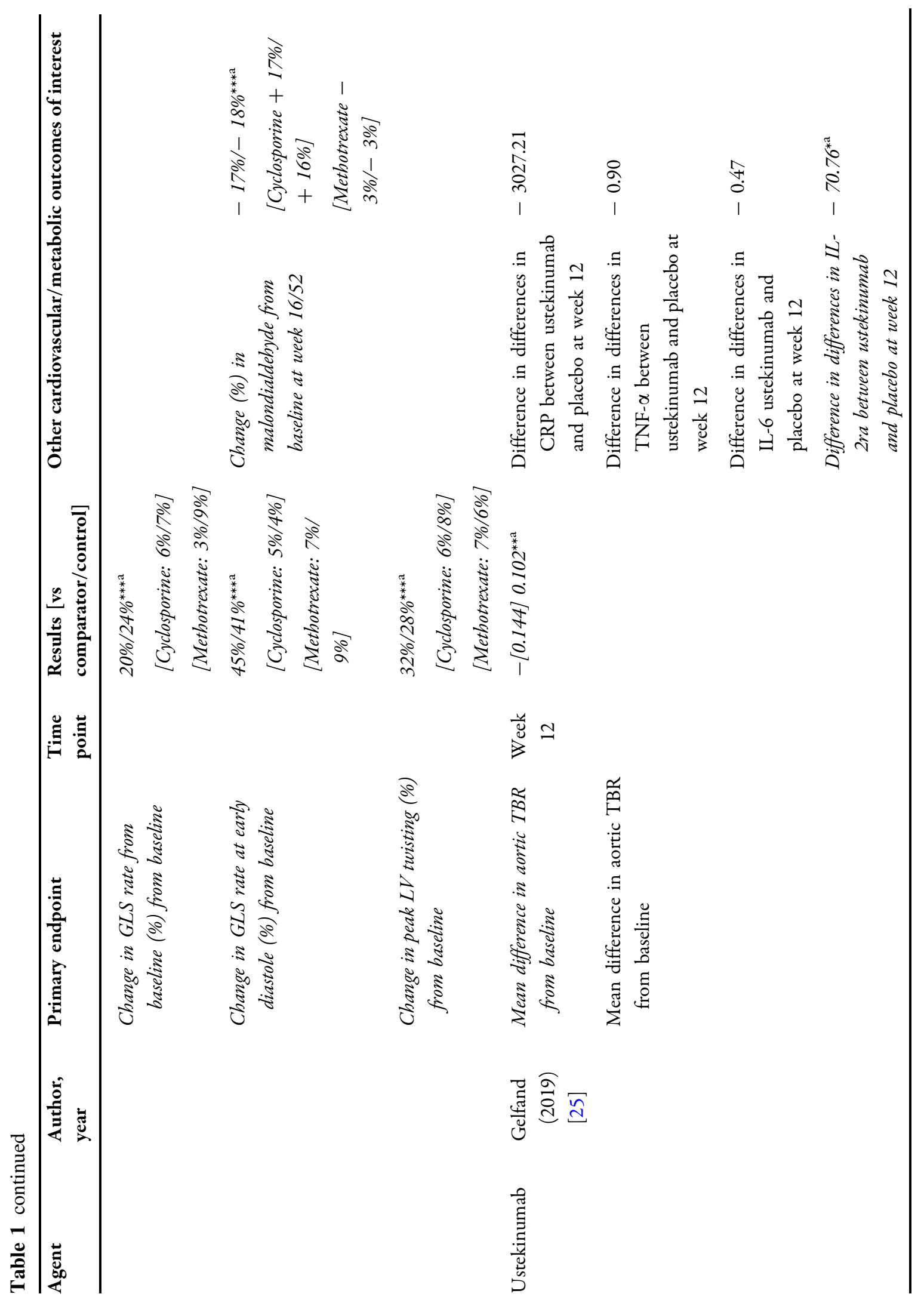




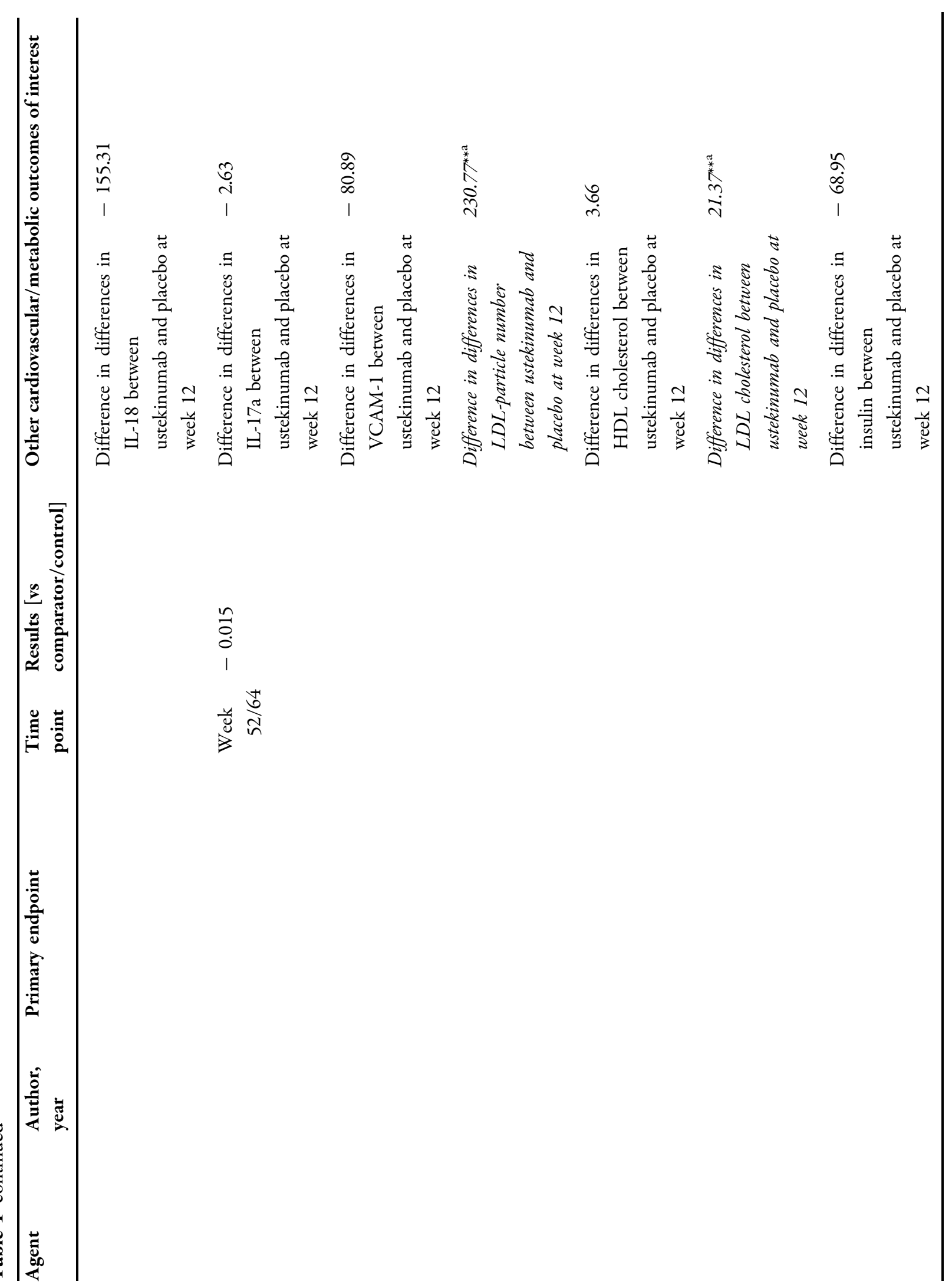




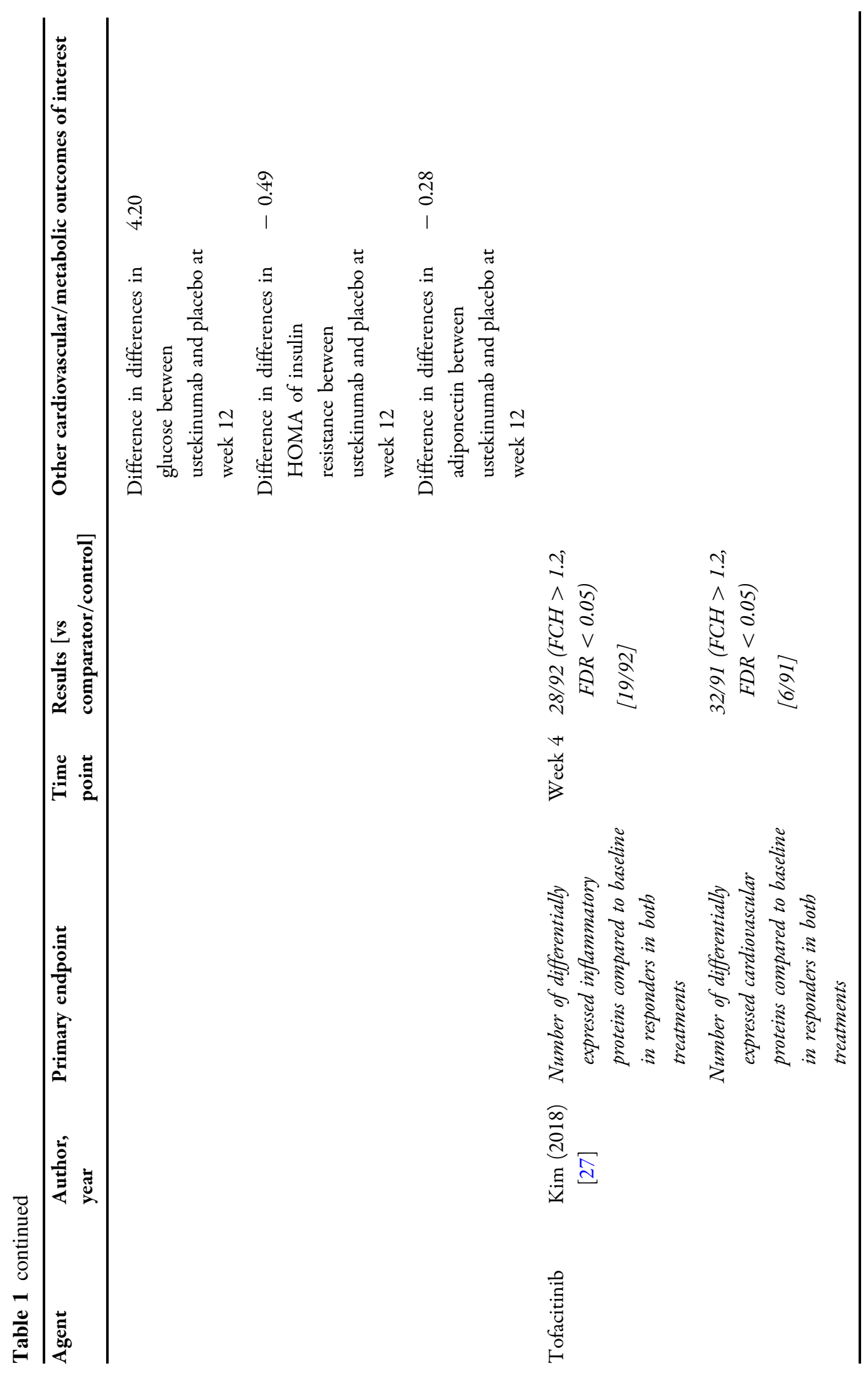




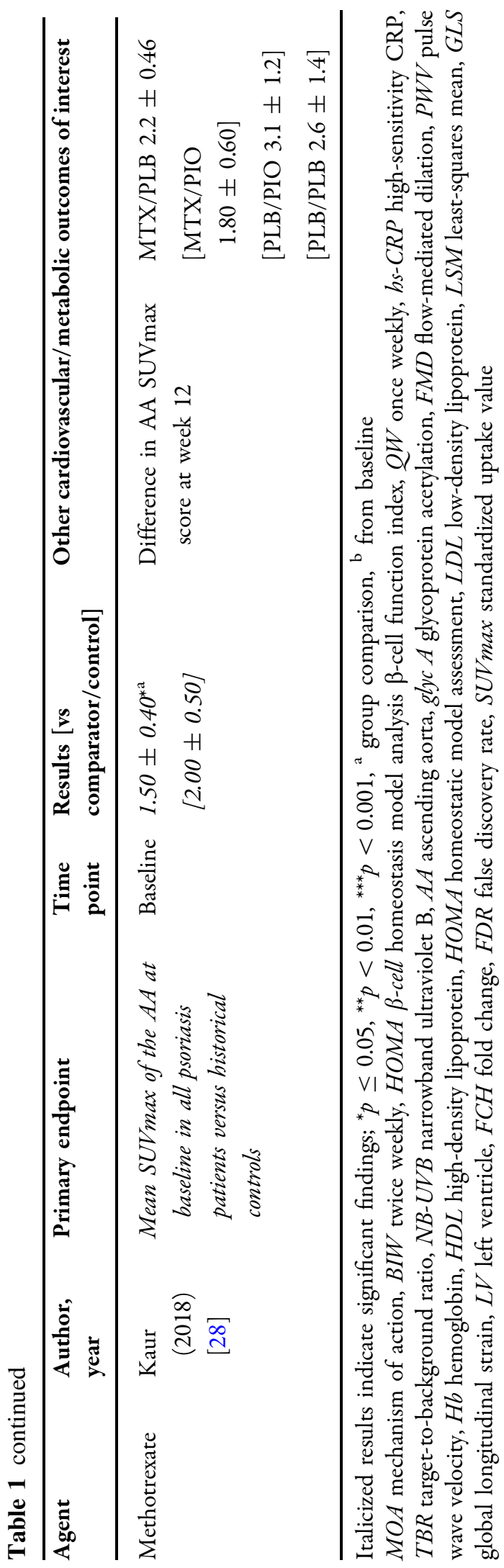

in psoriasis patients. One study evaluated how treatment with TNF- $\alpha$ inhibitors in psoriatic patients with type II diabetes impacts insulin resistance and insulin sensitivity [11]. Seventy psoriasis patients were randomized to receive treatment for 24 weeks with either TNF- $\alpha$ inhibitors (including etanercept, adalimumab, and infliximab) or a control (consisting of treatment with topicals, cyclosporine, or methotrexate). When compared to the control group, patients treated with TNF- $\alpha$ inhibitors showed a significant decrease from baseline in both fasting plasma glucose $[-2.74$ vs. $-0.02(p<0.01)]$ and hemoglobin A1c $[-1.3 \%$ vs. $0.2 \%$ $(p<0.01)]$ values. Treatment with TNF- $\alpha$ inhibitors also led to a significantly greater change in mean insulin resistance, which was assessed using the homeostasis model assessment (HOMA), when compared to control patients treated with other agents $[1.2$ vs. -0.3 $(p<0.01)]$.

\section{Etanercept}

Treatment with etanercept has demonstrated variable cardiovascular benefits in randomized clinical trials. An analysis of previously frozen patient blood from a randomized, double-blind, placebo-controlled study evaluated the effect of etanercept on C-reactive protein (CRP), which is a contributor to atherosclerosis and a predictor of coronary artery disease [12]. For the first 12 weeks, patients with psoriasis and psoriatic arthritis were randomized to receive either varying dosages of etanercept $(25 \mathrm{mg}$ once weekly, $25 \mathrm{mg}$ twice weekly, or $50 \mathrm{mg}$ twice weekly) or placebo. From week 12 to week 24, patients initially on placebo received etanercept $25 \mathrm{mg}$ twice weekly. Treatment with etanercept for 12 weeks resulted in significantly greater reductions in median CRP from baseline in psoriasis patients with and without psoriatic arthritis compared to placebo [2.7 $\mathrm{mg}$ vs. $0.1 \mathrm{mg}$ and $1.0 \mathrm{mg}$ vs. $0.1 \mathrm{mg}$, respectively $(p<0.001)]$. Placebo patients were able to achieve comparable results in CRP reduction after 12 weeks of etanercept therapy at week 24 .

The effect of etanercept treatment on metabolic parameters and risk factors has also been evaluated. A double-blind randomized pilot study treated 12 psoriasis patients with two or 
more risk factors for type 2 diabetes (in accordance with the American Diabetes Association) with either etanercept $25 \mathrm{mg}$ twice weekly or placebo for 2 weeks [13]. While the etanercept group did have a significant decrease in fasting serum insulin levels from baseline [baseline: $146 \pm 117$ vs. week 2 : $111 \pm 87 \quad(p=0.04)$ ], there was no significant difference in insulin sensitivity or secretion. Furthermore, treatment with etanercept did not significantly impact any of the other metabolic characteristics, including fasting serum glucose, cholesterol, or uric acid.

A more recent study with a longer treatment duration assessed the impact of etanercept on cardiometabolic biomarkers, including cholesterol, plasma insulin, quantitative insulin sensitivity check index (QUICKI), apolipoprotein (APO), and high-sensitivity CRP (hs-CRP) [14]. Plaque psoriasis patients were randomized double blind to etanercept $50 \mathrm{mg}$ once or twice weekly for 12 weeks. Following 12 weeks of treatment with etanercept once and twice weekly, statistically significant mean percent changes from baseline were observed in several biomarkers, including QUICKI $(-2.2 \%$ and $-2.7 \%$, respectively), Apo A1 (3.2\% and 2.8\%), Apo B:Apo A1 ratio ( $-3.5 \%$ and $-4.6 \%)$, and hs-CRP $(-65.5 \%$ and $-74.4 \%)(p<0.05)$. In addition, etanercept twice weekly resulted in significant mean percent changes in fasting plasma insulin (15.9\%) and high-density lipoprotein cholesterol (HDL-C, 2.9\%) from baseline at week $12(p<0.05)$.

\section{Adalimumab}

Adalimumab has demonstrated mixed efficacy in reducing cardiovascular and metabolic risk factors. Two studies demonstrated that treatment with adalimumab resulted in no significant improvement in vascular inflammation of the aorta or carotid arteries $[15,16]$. One randomized, double-blind, placebo-controlled study evaluated 107 psoriasis patients randomized to treatment with either adalimumab for 52 weeks or placebo for 16 weeks followed by adalimumab for 52 weeks [15]. Vascular inflammation was measured using positron emission tomography-computed tomography (PET/CT) with an injection of radiolabeled ${ }^{18} \mathrm{~F}$ fluoro-2-deoxy-D-glucose $\left({ }^{18} \mathrm{~F}-\mathrm{FDG}\right)$, which accumulates in the macrophage-dense atherosclerotic plaques in inflamed vessel walls. FDG uptake was then quantified via the vessel wall target-to-background ratio (TBR), providing a noninvasive measure of arterial plaque inflammation [17]. No significant differences in aortic or carotid artery TBR were demonstrated after 16 weeks of treatment with adalimumab. In fact, TBR in the carotid arteries increased from baseline after 52 weeks of adalimumab $(p=0.046)$. However, 16 weeks of treatment with adalimumab resulted in a statistically significant decrease from baseline in hs-CRP when compared to placebo [- $28.67 \%$ vs. $1.09 \%$, respectively $(p=0.012)]$.

The second study, which confirmed those findings, was a randomized, double-blind, placebo-controlled trial comparing the effects of adalimumab, narrowband ultraviolet B (NBUVB) phototherapy, and placebo on vascular inflammation of the ascending aorta and metabolic biomarkers [16]. Following the randomization phase (12 weeks), all patients either continued or started on adalimumab for a total of 52 weeks of treatment. After 12 weeks of treatment with adalimumab and NB-UVB, there was no significant change in maximum TBR compared to placebo [change compared to placebo: $\quad 0.64 \% \quad(p=0.795) \quad$ and $-1.60 \%$ $(p=0.540)$, respectively]. NB-UVB did result in a significantly decreased TBR compared to baseline [change compared to baseline: $-4.09 \%$ $(p=0.031)]$. Treatment with 52 weeks of adalimumab resulted in a significant decrease in TBR when compared to the absolute study baseline, but not to the adalimumab treatment baseline $[-3.80 \%(p=0.005)$ and $0.02 \%(p=0.987)$, respectively]. Following 12 weeks of treatment, both adalimumab and phototherapy resulted in significant decreases in serum CRP and IL-6 when compared to placebo $(p<0.05)$. Furthermore, treatment with adalimumab for 12 weeks also resulted in significant reductions in TNF- $\alpha$, a key proinflammatory cytokine, and glycoprotein acetylation (GlycA), which is positively associated with risk of death and major cardiovascular events [vs. placebo $(p<0.01)$ ] [18]. Following 52 weeks of treatment with adalimumab, significant improvements were noted in the biomarker levels of TNF- $\alpha$, GlycA, and 
CRP, but significantly negative impacts were seen in both IL-6 and high-density lipoprotein particle (HDL-P) levels $(p<0.05)$. Neither treatment had a significant impact on serum glucose metabolism markers.

\section{IL-17 inhibitors}

\section{Secukinumab}

Several studies have evaluated the impact of secukinumab on cardiovascular disease parameters such as endothelial dysfunction, vascular inflammation, and myocardial deformation. A randomized, double-blind, placebo-controlled study evaluated the impact of secukinumab versus placebo on flow-mediated dilation (FMD), which is a measure of vascular endothelial function and an early predictor of future cardiovascular events $[19,20]$. In total, 151 patients were randomized to either secukinumab $150 \mathrm{mg}$ or $300 \mathrm{mg}$ for 52 weeks or placebo for 12 weeks followed by one of the two secukinumab doses until week 52. Although no differences in FMD were noted at week 12 between secukinumab groups and placebo, significant improvements in FMD from baseline were present in both the secukinumab $150 \mathrm{mg}$ and secukinumab $300 \mathrm{mg}$ groups after 52 weeks of treatment $[+2.1 \%(p=0.0034)$ and $+2.1 \%$ $(p=0.0022)$, respectively]. Additionally, when compared to placebo at week 12 and baseline at week 52, secukinumab treatment was associated with a decrease in adiponectin, a biomarker that is suggested to be protective against the development of hypertension, insulin resistance, and cardiovascular disease $(p<0.05)$ [21]. No other clinically relevant changes were demonstrated in arterial stiffness, total plaque burden of the carotid artery or aorta, or serum biomarkers of systemic inflammation, lipids, or glucose metabolism.

The impact of secukinumab on vascular inflammation via ${ }^{18} \mathrm{~F}-\mathrm{FDG}$ PET/CT scans and serum cardiometabolic biomarkers was evaluated in a randomized, double-blind, placebocontrolled study of psoriasis patients [22]. Patients were randomized to either secukinumab $300 \mathrm{mg}$ or placebo for the first 12 weeks. Following this, placebo patients were switched to secukinumab for the remainder of the 52-week study. Evaluation with ${ }^{18}$ F-FDG PET/CT scans revealed no significant changes in TBR of the aorta following secukinumab treatment. Furthermore, no significant differences at week 12 in any markers of inflammation, insulin resistance, diabetic predictors, or adiposity were noted. At week 52, treatment with secukinumab was associated with a significant reduction from baseline in TNF- $\alpha(p=0.0063)$ and ferritin $(p=0.035)$, a biomarker positively associated with cardiovascular risk factors and occurrence of insulin resistance [23].

Further investigation demonstrated the effects of secukinumab on vascular and left ventricular (LV) function in patients with plaque psoriasis and psoriatic arthritis [24]. In the 1-year study, 100 psoriatic patients were randomized to receive secukinumab or cyclosporine, and 50 psoriatic patients who were beginning treatment with methotrexate served as control. Secukinumab resulted in greater improvements in LV myocardial function (LV global longitudinal strain, global longitudinal strain rate, global longitudinal strain rate at early diastole, and LV twisting), arterial elasticity (pulse wave velocity), and coronary flow reserve when compared to both cyclosporine and methotrexate. Oxidative stress, as measured by malondialdehyde and protein carbonyl levels, was also significantly improved after 1 year of secukinumab treatment $(p=0.03$ and $p=0.02$, respectively). However, cyclosporine was associated with a negative effect on oxidative stress markers $(p<0.05)$ and on arterial elasticity, as measured by pulse wave velocity [month 4: $+11 \%$, month $12:+14 \%$ $(p=0.02)]$.

\section{IL-12/23 inhibitors}

\section{Ustekinumab}

The effect of ustekinumab on aortic vascular inflammation was evaluated via TBR with ${ }^{18} \mathrm{~F}$ FDG PET/CT scans and biomarkers of systemic inflammation, lipids, and glucose metabolism in a phase IV, randomized, double-blind, placebo-controlled trial [25]. In total, 43 patients were randomized to either ustekinumab for 
52 weeks or placebo for 12 weeks followed by ustekinumab for an additional 52 weeks. At week 12 , psoriasis patients treated with ustekinumab were found to have a statistically significant $(18.65 \%)$ reduction in aortic vascular inflammation compared to placebo $(p=0.001)$, although the improvement was transient. At week 52 of treatment, ustekinumab demonstrated a neutral effect on vascular inflammation in both groups when compared to baseline. Ustekinumab treatment was also associated with several statistically significant changes to serum biomarkers at week 12 when compared to placebo. Decreases in vascular cell adhesion molecule 1, a mediator in the development of atherosclerosis, and IL-2 receptor $\alpha$, which is positively associated with cardiovascular mortality and incident stroke and heart failure, were noted at week 12 [vs. placebo $(p<0.05)$ ] [26]. However, ustekinumab treatment for 12 weeks also resulted in increases in LDL and several other apolipoprotein-B lipoproteins [vs. placebo $(p<0.05)]$. Following 52 weeks of treatment, both ustekinumab treatment groups had significant decreases in IL-1 $\beta$, IL-17a, and IL-18 and significant increases in IL-12/23, high-density lipoprotein particle size (hdl-z), large verylow-density lipoprotein particle number (vldl$p)$, and leptin compared to baseline $(p<0.05)$.

\section{Other systemic agents}

\section{Tofacitinib}

The impact of tofacitinib versus etanercept on inflammatory and cardiovascular proteins was investigated with archived blood samples from 266 psoriasis patients in a randomized, doubleblind, phase III psoriasis trial [27]. Following 4 weeks of treatment with tofacitinib and etanercept, IL-6, CCL20, and CXCL10, which are reported to be common inflammatory molecules in both psoriasis and atherosclerosis, were reduced [fold change $(\mathrm{FCH})>1.2$ and false discovery rate $(\mathrm{FDR})<0.05]$. Furthermore, only tofacitinib responders who achieved PASI 75 demonstrated a significant reduction in some psoriasis-associated cardiovascular proteins, including CHI3L1, E-selectin, hK11, IL-16, TNF receptor 1, TNF-related activation-induced cytokine, and matrix metalloproteinase-12 $(\mathrm{FCH}>1.2$ and FDR $<0.05)$.

\section{Methotrexate}

While methotrexate served as a control for two of the abovementioned studies [11, 24], it was also studied in a randomized, double-blind, placebo-controlled trial evaluating vascular inflammation as measured by ${ }^{18} \mathrm{~F}$-FDG PET/CT [28]. A total of 15 psoriasis patients were randomized to four different treatments: methotrexate, methotrexate and pioglitazone, pioglitazone, or placebo. Vascular inflammation of the ascending aorta was higher in psoriasis patients at baseline compared to historical controls. However, no significant difference in aortic vascular inflammation, which was calculated through the maximum standardized uptake value of FDG in the arterial wall, was observed in any of the treatment groups following 12 weeks of treatment.

\section{DISCUSSION}

Cardiovascular and metabolic comorbidities pose a significant burden to psoriasis patients. The presence of cardiovascular comorbidity in psoriasis patients is associated with lower health-related quality of life with significant decreases in physical and mental health $(p<0.01)$ [8]. Additionally, psoriasis patients with cardiovascular comorbidity reported greater rates of presenteeism, which is the loss of productivity in the workplace that occurs due to medical conditions, and overall work and activity impairment than psoriasis patients without cardiovascular disease $(p<0.05)$ [8].

Cardiometabolic comorbidity is also associated with greater healthcare utilization and costs. Accompanying cardiovascular disease in psoriasis patients was associated with 1.9 times more health care visits and 2-3 times more hospitalizations within the previous 6 months $(p<0.05)$ [8]. Another study demonstrated that patients with cardiovascular disease had an incidence rate ratio of $2.3(2.2-2.5)$ for emergency room visits, 2.6 (2.4-2.8) for hospitalizations, and 1.5 (1.4-1.5) for outpatient clinic visits [29]. Cardiovascular comorbidity also 
results in a considerable incremental economic burden. The presence of comorbid cardiovascular disease was associated with an estimated $\$ 8275$ in annual adjusted incremental costs over psoriasis patients without comorbid diseases [29].

This burden associated with cardiometabolic comorbidities disproportionately impacts younger patients. Studies demonstrate that the relative risk of myocardial infarction and cardiovascular mortality is highest among young patients with severe psoriasis [2, 30]. Overall, the presence of cardiometabolic comorbidities amplifies the already substantial psychological, physical, and economic burden associated with psoriasis, highlighting the need to explore shared therapeutic avenues.

Systemic agents have the potential to positively impact both cardiovascular and metabolic health due to the shared activation of systemic immune pathways between psoriasis, metabolic syndrome, and cardiovascular disease. The current data from randomized clinical trials demonstrate that biologic and other systemic agents have varying levels of impact in imaging and biomarkers of cardiometabolic disease. Differences also exist between the impact of systemic agents on cardiometabolic dysfunction, suggesting that some treatments may be more beneficial than others. While those studies do not evaluate concrete cardiometabolic or mortality outcomes, the parameters assessed are closely associated with overall cardiovascular and metabolic health.

Four clinical trials evaluated the impact of systemic agents on vascular inflammation through TBR via ${ }^{18}$ F-FDG PET/CT. Imaging with $\mathrm{CT} / \mathrm{PET}$ and injection of ${ }^{18} \mathrm{~F}-\mathrm{FDG}$ is a proposed noninvasive reflection of arterial inflammation [17]. Additionally, ${ }^{18}$ F-FDG PET/CT is a suggested surrogate marker of early coronary artery disease and a proposed predictor of future major cardiovascular events [17, 31]. Ustekinumab, adalimumab, and NB-UVB phototherapy were all associated with varying levels of improvement in vascular inflammation via ${ }^{18}$ F-FDG PET/CT, but only ustekinumab demonstrated a statistically significant benefit when compared to placebo. While these results are promising, several of these studies evaluated cardiovascular inflammation after $12-16$ weeks, a time period that may be too short to perceive real changes in the chosen imaging modalities.

Several studies also assessed clinical biomarkers of systemic inflammation with CRP, given the significant role it plays in promoting vascular inflammation and cardiovascular events [32]. CRP levels over $10 \mathrm{mg} / \mathrm{L}$ are associated with a greater than $4 \%$ risk of developing a fatal cardiovascular event in 10 years, suggesting that a reduction in CRP levels could represent a reduction in comorbid cardiovascular risk and events [32]. Specifically, etanercept, adalimumab, and NB-UVB were associated with significant reductions in CRP.

Other serum biomarkers, such as glucose, insulin, and cholesterol, were used to assess the impact of systemic agents on metabolic syndrome parameters. Metabolic syndrome, which is present in almost a third of psoriasis patients, is a significant predictor of cardiovascular disease [33]. Patients with metabolic syndrome have a 2.5-fold increased risk of myocardial infarction than patients without metabolic syndrome [34]. Treatment with etanercept resulted in varying levels of benefit across several metabolic parameters. However, there is still not enough data to characterize the clinical benefit, if any, of systemic agents. Although the endpoints evaluated in these randomized clinical studies are surrogate markers for disease outcomes, the results still suggest an overall positive impact on cardiovascular and metabolic health.

While the follow up of these studies was limited to 1 year or less, a long-term follow-up study revealed that improvements in biomarkers may be more than just transient changes in serum concentrations. A study of patients with moderate to severe psoriasis demonstrated that biomarkers associated with systemic inflammation, E-selectin, IL-22, and hs-CRP, were still significantly decreased 24 months after treatment with adalimumab [35]. This suggests that secondary improvements in inflammation associated with systemic therapy may persist with continuation of treatment.

Furthermore, the results from these randomized clinical trials are supported by recent prospective observational studies which 
demonstrate that biologic therapy is associated with improvements in cardiovascular disease parameters [36, 37]. Treatment with TNF- $\alpha$ inhibitors (adalimumab and etanercept), IL-12/ 23 inhibitor (ustekinumab), and IL-17 inhibitors (secukinumab and ixekizumab) was associated with a reduction in noncalcified coronary artery plaque when compared to patients who were not treated with biologics [37]. Additionally, a second prospective cohort study demonstrated that 1 year of biologic therapy was associated with a reduction in coronary artery inflammation [36].

Emerging data suggest that in addition to the secondary impact on cardiometabolic parameters, systemic treatment may also improve the life expectancy of psoriasis patients. The PSOLAR longitudinal study demonstrated that patients with moderate to severe psoriasis who underwent systemic therapy with biologics had a reduced risk of all-cause mortality and cardiovascular mortality when compared to those with no exposure to biologics [38]. Long-term treatment with methotrexate was also associated with a reduced risk of mortality.

Overall, the impact of systemic agents on the imaging and biomarkers of cardiometabolic disease is promising, and these findings highlight the potential added benefit of systemic therapy for psoriasis patients. While many factors currently dictate the choice of systemic therapies, including safety, efficacy, and patient preference, the secondary benefit of systemic agents for cardiometabolic parameters may play a role in the treatment algorithm of psoriasis therapy in the future. Additionally, given the disproportionate cardiometabolic burden placed on younger psoriasis patients, recognition of the increased cardiovascular risk and early intervention with appropriate treatment is critical. A discussion with young psoriasis patients regarding the potential benefit of sustained systemic therapy may be considered earlier in the disease course. These agents may also have an impact on future major cardiovascular events, but randomized placebo-controlled trials evaluating the direct impact of systemic agents on outcomes are needed to further define this benefit.

\section{CONCLUSION}

Cardiovascular and metabolic comorbidities are highly associated with psoriasis. Systemic inflammation, endothelial dysfunction, and pathologic angiogenesis link psoriasis with the development of cardiometabolic disorders. The shared inflammatory pathways indicate that systemic treatments may be able to target these domains concurrently. Randomized clinical trials of adalimumab, methotrexate, cyclosporine, etanercept, infliximab, secukinumab, tofacitinib, and ustekinumab revealed varying levels of benefit for cardiometabolic markers and risk factors. While the current data exhibit varying benefit in the simultaneous treatment of psoriasis and cardiometabolic risk factors and biomarkers, there is still a need for further randomized clinical trials that evaluate cardiometabolic outcomes to further characterize the added benefit of these systemic agents.

\section{ACKNOWLEDGEMENTS}

Funding. No funding or sponsorship was received for this study or publication of this article.

Authorship. All named authors meet the International Committee of Medical Journal Editors (ICMJE) criteria for authorship for this article, take responsibility for the integrity of the work as a whole, and have given their approval for this version to be published.

Author Contributions. Megan Mosca contributed to the concept, design, drafting, and editing of the manuscript. Julie Hong and Edward Hadeler contributed to the drafting and editing of the manuscript. Dr. Nicholas Brownstone, Dr. Marwa Hakimi, and Dr. Wilson Liao contributed to the concept and editing of the manuscript. Dr. Tina Bhutani contributed to the concept, design, drafting, and editing of the manuscript. All authors discussed the results and contributed to the final manuscript. 
Disclosures. Dr. Tina Bhutani has received research funding from Abbvie, Celgene, Galderma, Janssen, Pfizer, Regeneron, and Sun. She has served as an advisor for Abbvie, BoehringerIngelheim, Bristol Myers Squibb, Pfizer, Leo, Lilly, and Novartis. Dr. Wilson Liao has received research grant funding from Abbvie, Amgen, Janssen, Leo, Novartis, Pfizer, Regeneron, and TRex Bio. Megan Mosca, Julie Hong, Edward Hadeler, Dr. Marwa Hakimi and Dr. Nicholas Brownstone have no conflicts of interest to disclose.

Compliance with Ethics Guidelines. This article is based on previously conducted studies and does not contain any new studies with human participants or animals performed by any of the authors.

Open Access. This article is licensed under a Creative Commons Attribution-NonCommercial 4.0 International License, which permits any non-commercial use, sharing, adaptation, distribution and reproduction in any medium or format, as long as you give appropriate credit to the original author(s) and the source, provide a link to the Creative Commons licence, and indicate if changes were made. The images or other third party material in this article are included in the article's Creative Commons licence, unless indicated otherwise in a credit line to the material. If material is not included in the article's Creative Commons licence and your intended use is not permitted by statutory regulation or exceeds the permitted use, you will need to obtain permission directly from the copyright holder. To view a copy of this licence, visit http://creativecommons.org/licenses/by$\mathrm{nc} / 4.0 /$.

\section{REFERENCES}

1. World Health Organization. Global report on psoriasis. 2016. https://apps.who.int/iris/handle/ 10665/204417. Accessed 18 Apr 2021.

2. Gelfand JM, Neimann AL, Shin DB, Wang X, Margolis DJ, Troxel AB. Risk of myocardial infarction in patients with psoriasis. JAMA. 2006;296(14): 1735-41.

3. Lai YC, Yew YW. Psoriasis as an independent risk factor for cardiovascular disease: an epidemiologic analysis using a national database. J Cutan Med Surg. 2016;20(4):327-33.

4. Mansouri B, Kivelevitch D, Natarajan B, Joshi AA, Ryan C, Benjegerdes $\mathrm{K}$, et al. Comparison of coronary artery calcium scores between patients with psoriasis and type 2 diabetes. JAMA Dermatol. 2016;152(11):1244-53.

5. Armstrong AW, Harskamp CT, Armstrong EJ. Psoriasis and the risk of diabetes mellitus: a systematic review and meta-analysis. JAMA Dermatol. 2013;149(1):84-91.

6. Armstrong AW, Voyles SV, Armstrong EJ, Fuller EN, Rutledge JC. A tale of two plaques: convergent mechanisms of T-cell-mediated inflammation in psoriasis and atherosclerosis. Exp Dermatol. 2011;20(7):544-9.

7. Armstrong AW, Voyles SV, Armstrong EJ, Fuller EN, Rutledge JC. Angiogenesis and oxidative stress: common mechanisms linking psoriasis with atherosclerosis. J Dermatol Sci. 2011;63(1):1-9.

8. Augustin M, Vietri J, Tian H, Gilloteau I. Incremental burden of cardiovascular comorbidity and psoriatic arthritis among adults with moderate-tosevere psoriasis in five European countries. J Eur Acad Dermatol Venereol. 2017;31(8):1316-23.

9. Abuabara K, Azfar RS, Shin DB, Neimann AL, Troxel $\mathrm{AB}$, Gelfand JM. Cause-specific mortality in patients with severe psoriasis: a population-based cohort study in the UK. Br J Dermatol. 2010;163(3):586-92.

10. Wu JJ, Poon K-YT, Channual JC, Shen AY-J. Association between tumor necrosis factor inhibitor therapy and myocardial infarction risk in patients with psoriasis. Arch Dermatol. 2012;148(11): $1244-50$.

11. Al-Mutairi N, Shabaan D. Effects of tumor necrosis factor $\alpha$ inhibitors extend beyond psoriasis: insulin sensitivity in psoriasis patients with type 2 diabetes mellitus. Cutis. 2016;97(3):235-41.

12. Strober B, Teller C, Yamauchi P, Miller JL, Hooper $\mathrm{M}$, Yang Y-C, et al. Effects of etanercept on C-reactive protein levels in psoriasis and psoriatic arthritis. Br J Dermatol. 2008;159(2):322-30.

13. Martínez-Abundis E, Reynoso-von Drateln C, Hernández-Salazar E, González-Ortiz M. Effect of etanercept on insulin secretion and insulin sensitivity in a randomized trial with psoriatic patients at risk for 
developing type 2 diabetes mellitus. Arch Dermatol Res. 2007;299(9):461-5.

14. Puig L, Strohal R, Fuiman J, Pedersen R, Szumski A, Koenig AS, et al. Cardiometabolic biomarkers in chronic plaque psoriasis before and after etanercept treatment. J Dermatolog Treat. 2014;25(6):470-81.

15. Bissonnette R, Harel F, Krueger JG, Guertin M-C, Chabot-Blanchet $M$, Gonzalez J, et al. TNF- $\alpha$ antagonist and vascular inflammation in patients with psoriasis vulgaris: a randomized placebo-controlled study. J Invest Dermatol. 2017;137(8): $1638-45$.

16. Mehta NN, Shin DB, Joshi AA, Dey AK, Armstrong AW, Duffin KC, et al. Effect of 2 psoriasis treatments on vascular inflammation and novel inflammatory cardiovascular biomarkers: a randomized placebocontrolled trial. Circ Cardiovasc Imaging. 2018;11(6): e007394.

17. Emami H, Tawakol A. Noninvasive imaging of arterial inflammation using FDG-PET/CT. Curr Opin Lipidol. 2014;25(6):431-7.

18. An L, Liu Q, Feng H, Bai X, Dang Y, Li C, et al. Increased glycoprotein acetylation is associated with high cardiac event rates: analysis using coronary computed tomography angiography. Anatol J Cardiol. 2018;20(3):152-8.

19. von Stebut E, Reich K, Thaçi D, Koenig W, Pinter A, Körber A, et al. Impact of secukinumab on endothelial dysfunction and other cardiovascular disease parameters in psoriasis patients over 52 weeks. J Investig Dermatol. 2019;139(5):1054-62.

20. Inaba Y, Chen JA, Bergmann SR. Prediction of future cardiovascular outcomes by flow-mediated vasodilatation of brachial artery: a meta-analysis. Int J Cardiovasc Imaging. 2010;26(6):631-40.

21. Shibata R, Ouchi N, Murohara T. Adiponectin and cardiovascular disease. Circ J. 2009;73(4):608-14.

22. Gelfand JM, Shin DB, Duffin KC, Armstrong AW, Blauvelt A, Tyring SK, et al. A randomized placebocontrolled trial of secukinumab on aortic vascular inflammation in moderate-to-severe plaque psoriasis (VIP-S). J Investig Dermatol. 2020;140(9):17841793.e2.

23. Liu J-R, Liu Y, Yin F-Z, Liu B-W. Serum ferritin, an early marker of cardiovascular risk: a study in Chinese men of first-degree relatives with family history of type 2 diabetes. BMC Cardiovasc Disord. 2019;19(1):82.

24. Makavos G, Ikonomidis I, Andreadou I, Varoudi M, Kapniari I, Loukeri E, et al. Effects of interleukin 17A inhibition on myocardial deformation and vascular function in psoriasis. Can $\mathrm{J}$ Cardiol. 2020;36(1):100-11.

25. Gelfand JM, Shin DB, Alavi A, Torigian DA, Werner $\mathrm{T}$, Papadopoulos $\mathrm{M}$, et al. A phase IV, randomized, double-blind, placebo-controlled crossover study of the effects of ustekinumab on vascular inflammation in psoriasis (the VIP-U Trial). J Invest Dermatol. 2020;140(1):85-93.e2.

26. Durda P, Sabourin J, Lange EM, Nalls MA, Mychaleckyj JC, Jenny NS, et al. Plasma levels of sIL-2R $\alpha$ : associations with clinical cardiovascular events and genome-wide association scan. Arterioscler Thromb Vasc Biol. 2015;35(10):2246-53.

27. Kim J, Tomalin L, Lee J, Fitz LJ, Berstein G, Correada Rosa J, et al. Reduction of inflammatory and cardiovascular proteins in the blood of patients with psoriasis: differential responses between tofacitinib and etanercept after 4 weeks of treatment. J Invest Dermatol. 2018;138(2):273-81.

28. Kaur S, Shafiq N, Dogra S, Mittal BR, Attri SV, Bahl A, et al. 18F-fluorodeoxyglucose positron emission tomography-based evaluation of systemic and vascular inflammation and assessment of the effect of systemic treatment on inflammation in patients with moderate-to-severe psoriasis: a randomized placebo-controlled pilot study. Indian J Dermatol Venereol Leprol. 2018;84(6):660-6.

29. Feldman SR, Tian H, Gilloteau I, Mollon P, Shu M. Economic burden of comorbidities in psoriasis patients in the United States: results from a retrospective U.S. database. BMC Health Serv Res. 2017;17(1):337.

30. Mallbris L, Akre O, Granath F, Yin L, Lindelöf B, Ekbom A, et al. Increased risk for cardiovascular mortality in psoriasis inpatients but not in outpatients. Eur J Epidemiol. 2004;19(3):225-30.

31. Joshi AA, Lerman JB, Dey AK, Sajja AP, Belur AD, Elnabawi YA, et al. Association between aortic vascular inflammation and coronary artery plaque characteristics in psoriasis. JAMA Cardiol. 2018;3(10):949-56.

32. Cozlea DL, Farcas DM, Nagy A, Keresztesi AA, Tifrea $\mathrm{R}$, Cozlea $\mathrm{L}$, et al. The impact of $\mathrm{C}$ reactive protein on global cardiovascular risk on patients with coronary artery disease. Curr Health Sci J. 2013;39(4):225-31.

33. Rodríguez-Zúñiga MJM, García-Perdomo HA. Systematic review and meta-analysis of the association between psoriasis and metabolic syndrome. J Am Acad Dermatol. 2017;77(4):657-666.e8.

34. Mente A, Yusuf S, Islam S, McQueen MJ, Tanomsup $\mathrm{S}$, Onen $\mathrm{CL}$, et al. Metabolic syndrome and risk of 
acute myocardial infarction: a case-control study of 26,903 subjects from 52 countries. J Am Coll Cardiol. 2010;55(21):2390-8.

35. Gkalpakiotis S, Arenbergerova M, Gkalpakioti P, Potockova J, Arenberger P, Kraml P. Long-term impact of adalimumab therapy on biomarkers of systemic inflammation in psoriasis: results of a 2 year study. Dermatol Therapy. 2020;33(6): e14110.

36. Elnabawi YA, Oikonomou EK, Dey AK, Mancio J, Rodante JA, Aksentijevich M, et al. Association of biologic therapy with coronary inflammation in patients with psoriasis as assessed by perivascular fat attenuation index. JAMA Cardiol. 2019;4(9): 885-91.
37. Elnabawi YA, Dey AK, Goyal A, Groenendyk JW, Chung JH, Belur AD, et al. Coronary artery plaque characteristics and treatment with biologic therapy in severe psoriasis: results from a prospective observational study. Cardiovasc Res. 2019;115(4): 721-8.

38. Langley RG, Poulin Y, Srivastava B, Lafferty KP, Fakharzadeh S, Langholff W, et al. Reduced risk of mortality associated with systemic psoriasis treatment in the Psoriasis Longitudinal Assessment and Registry (PSOLAR): a nested case-control analysis. J Am Acad Dermatol. 2021;84(1):60-9. 